? Die gesellschaftlichen und schulpolitischen Rahmenbedingungen für Legastheniker haben sich geändert. Würden Sie rückblickend sagen, dass die Schwierigkeiten Betroffener heute früher als Störung erkannt werden und die Legasthenie im Schulbetrieb eher akzeptiert wird?

Soremba: Jein: die höheren Schulen sind oft aufgeschlossener als die Grundschule. Dabei ist diese ja verantwortlich für so vieles: Sie prägt die Lernbiografie - was in dieser Zeit versäumt wird, ist kaum wieder gutzumachen. Die Gymnasien arbeiten meistens ganz gut mit uns zusammen, wenn wir betroffenen Kindern ein Gutachten erstellen und darum bitten, ihnen Notenschutz oder Nachteilsausgleich zu gewähren.

? Was treibt Sie an?

Soremba: Für mich steht das Individuum mit seinen Nöten und Qualen im Mittelpunkt. Nach meiner Grundüberzeugung kann und darf nicht sein, dass so ein kleiner Mensch, der sich auf die Schule gefreut hat, auf einmal lernen muss, dass er ein Versager ist und unter Tränen und Seelenqualen lesen und schreiben erlernen muss! Das Selbstbewusstsein eines Kindes muss erhalten bzw. gestärkt werden - wie das eines jeden Menschen.

Oder denken Sie nur einmal an den Analphabetismus: Geschätzte vier Millionen Deutsche können nicht richtig lesen und schreiben! Viele glauben ja gar nicht, dass es das bei uns gibt.

Die Betroffenen leiden unter Schamgefühl, Ausgrenzung und Hoffnungslosigkeit. In meinem Beratungsdienst, wo sie keinen Namen zu nennen brauchen, öffnen sich viele in ihrer Seelennot.

? Was wollen Sie den Lesern in Ihrem neuen Buch zum Thema "Lesen lernen vor dem Schuleintritt"vermitteln?

Soremba: Ich möchte den Eltern sagen: Ein Kind lernt vom ersten Augenblick seines Lebens an, und was wir am Anfang versäumen, ist nachher gar nicht wieder gutzumachen! Kinder können im frühesten Alter der Sprachentwicklung das Lesen erlernen, und Lesenlernen ist entscheidend wichtig für die Entwicklung von Intelligenz und Begabung. Die Fähigkeit, zu lesen, ist die Grundlage allen höheren Lernens. Je früher wir also den Kindern Augen und Sinne zum Lesen öffnen, umso besser.

Ein Kind hat ja von Beginn an optische Lerneindrücke. Man hat herausgefunden, dass schon Säuglinge in der Lage sind, ganz bestimmte, große Schriftbilder zu erfassen, zu speichern und wieder abzurufen - nur soviel vorab zu diesen neuen Forschungserkenntnissen. Mir geht es darum, dass die Kinder mit Freude an das herangebracht werden, was ihnen später im Leben möglicherweise hilft. Wesentlich ist natürlich, dass dies mit Liebe und ohne Drill geschieht.

! Frau Soremba, vielen Dank für das Gespräch.

\section{Das Interview führte Waltraud} Paukstadt.

\title{
Sprachtest jetzt auch für Dreijährige
}

Der Kurztest zur Sprachbeurteilung von zweijährigen Kindern durch die Eltern, SBE-2-KT, wurde nun durch den SBE-3-KT für die Dreijährigen ergänzt. Die Fragebogentests wurden an der Poliklinik für Kinder- und Jugendpsychiatrie der Ludwig Maximilians Universität München entwickelt und können unter den folgenden Webadressen kostenlos aus dem Internet heruntergeladen werden:

\section{www.kjp.med.uni-muenchen.de/sprachstoerungen/SBE-2-KT.php} www.kjp.med.uni-muenchen.de/sprachstoerungen/SBE-3-KT.php

Auch wenn das IOWiG kürzlich den Nutzen einer frühen Diagnose und Therapie von Sprachentwicklungsverzögerungen mangels vorliegender Studien bezweifelte, sind dem Kinderarzt durch die beiden Tests Instrumente an die Hand gegeben, die eine genaue Einschätzung und Verlaufsbeurteilung der Sprachentwicklung wesentlich erleichtern. 